\title{
Imaging the COVID-19: a practical guide
}

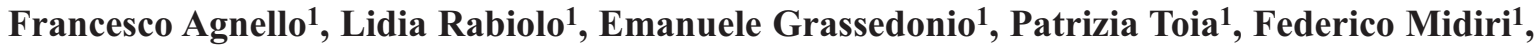 \\ Luigi Spatafora $^{1}$, Francesco Matteini ${ }^{1}$, Lorenzo Tesè ${ }^{2}$, Ludovico La Grutta ${ }^{1}$, Massimo Galia ${ }^{1}$ \\ ${ }^{1}$ Department of Radiology, Policlinico "Paolo Giaccone", University of Palermo; ${ }^{2}$ Department of Radiology, Azienda \\ Ospedali Riuniti Villa Sofia-Cervello, Palermo, Italy
}

\begin{abstract}
The Coronavirus Disease 2019 (COVID-19) represents the first medical catastrophe of the new millennium. Although imaging is not a screening test for COVID-19, it plays a crucial role in evaluation and follow-up of COVID-19 patients. In this paper, we will review typical and atypical imaging findings of COVID-19.
\end{abstract}

\section{Introduction}

The Coronavirus Disease 2019 (COVID-19) represents the first medical catastrophe of the new millennium. COVID-19 initially presented in China, in the Wuhan area, Hubei province, at the end of 2019, and quickly spreads around the globe because of its easy human-to-human transmission, hospital infection, and international air travel [1-4]. At the time of this writing, more than 34 million confirmed cases, and more than one million of deaths were reported globally by the World Health Organization (WHO) [5]. COVID-19

\footnotetext{
Correspondence: Emanuele Grassedonio, Department of Radiology, University Hospital Paolo Giaccone, University of Palermo, Via del Vespro 127, 90100 Palermo, Italy.

Tel. +39.091.6552356 - Fax: +39.091.6552324

E-mail: egrassedonio@gmal.com
}

Key words: COVID-19; CT; COVID-19 pneumonia.

Authors' contributions: All the authors made a substantive intellectual contribution. All the authors have read and approved the final version of the manuscript and agreed to be accountable for all aspects of the work.

Conflict of interest: The authors declare that they have no competing interests, and all authors confirm accuracy.

Received for publication: 2 October 2020.

Accepted for publication: 17 January 2021.

${ }^{\circ}$ Copyright: the Author(s), 2021

Licensee PAGEPress, Italy

Monaldi Archives for Chest Disease 2021; 91:1630

doi: 10.4081/monaldi.2021.1630

This article is distributed under the terms of the Creative Commons Attribution Noncommercial License (by-nc 4.0) which permits any noncommercial use, distribution, and reproduction in any medium, provided the original author(s) and source are credited. is due to an aggressive, highly contagious, betacoronavirus that involves mainly the respiratory system. Fever, dry cough, and dyspnea are the most common symptoms, while rhinorrhea and sneezing are less frequent [6]. This suggests that the target cells are preferentially located in the lower airways [6]. Less frequently, patients present central nervous system or gastrointestinal symptoms [6]. The absence of clinical symptoms was reported in approximately $50 \%$ of patients at the time of first diagnosis [7].

Individuals with old age, obesity, or other comorbidities are at increased risk to develop pneumonia, acute respiratory distress syndrome, and multiorgan failure suggesting that COVID-19 pathogenicity can depend on patient immune response $[8,9]$. Because of the absence of proven drugs and vaccines, early diagnosis, timely treatment, and immediate patient isolation are the pillars for containing the infection. The gold standard for diagnosis is positive results of real-time reverse transcription-polymerase chain reaction (RT-PCR) test of swabs taken from the nasopharynx and/or oropharynx $[3,10]$. However, a high rate of false-negative RT-PCR results is reported due to multiple causes, such as insufficient cellular material for detection and improper extraction of nucleic acids from clinical materials $[11,12]$. This leads to a delay in diagnosis and treatment, and a spread of the disease. Imaging can flag suspicious cases and alert referring physicians. In this paper we review the role of imaging in COVID-19 patients.

\section{Chest findings}

Chest CT is the main imaging methodology for detecting pulmonary involvement and optimized the future treatment because of high sensitivity and speed [13-15]. Although CT is not a screening test for COVID-19, it is strongly recommended for initial evaluation and follow-up of COVID-19 positive patients and to exclude other disease conditions, which can be treated (e.g., pulmonary embolism) $[10,16,17]$. Other imaging tests such as chest radiography (CXR) and lung ultrasound (LUS) are not routinely recommended in clinal practice.

CXR has low sensitivity for the detection of COVID-19 pneumonia in early and mild stages [8]. Currently, CXR is indicated in severe and critical COVID-19 patients, which require bedside exam, and in resource-constrained environments [18].

LUS has been proposed as an alternative tool to quickly assess lung injury. LUS can identify alteration in subpleural regions, which represents the typical COVID-19 locations [19]. Similar to CXR, LUS has some utility in bedside in critical patients [20]. Almost all COVID-19 confirmed patients show typical CT features including bilateral ground glass opacities (GGO), consolidation, vascular enlargement, and crazy-paving pattern. Most abnormalities are bilateral and involve more segments with a predomi- 
nant peripheral and posterior distribution and a slight predominance of lower lobes $[21,22]$. During the course of disease nodule, reversed halo sign, pleural changes, pericardial effusion, and enlargement of hilar and mediastinal lymphadenopathies can also occur. Infection control and regression of CT abnormalities can lead to tissue organization and fibrosis [23,24]. Extra-pulmonary abnormalities are absent in early stages and can occur in severe type of disease [24]. Unfortunately, CT findings of COVID-19 are not specific and overlap with those of other viral and not viral pneumonia [22,25-27]. Moreover, CT features are not always consistent with the clinical stage. Specifically, some asymptomatic patients can show typical CT features and some patients with clinically severe disease can show normal CT [28].

\section{Ground glass opacity}

GGO represents the most common CT finding in COVID-19 patients, with a reported incidence of 49 to $94 \%$ [29]. GGO is an area of increased attenuation with the preservation of bronchial and vascular margins [30]. In the context of COVID-19, GGO results from by partial filling of airspaces and interstitial thickening [30]. Three patterns of GGO have been described: pure GGO, GGO with superimposed intra and interlobular septal thickening ("crazy paving"), and GGO with consolidation [22] (Figure 1). Different patterns can often be seen simultaneously in the same patient [22]. Pure GGO and GGO with consolidation are the most common patterns in early stage of the disease $[23,24]$. Of note, GGO with peripheral distribution and unilateral location is the most common abnormality in asymptomatic (so called covert transmitter), patients.

\section{Consolidation}

Consolidation is a patchy area of increased attenuation, which renders the lung solid (Figure 2) [30]. Unlike GGO, the filling of airspaces is complete and obscures totally bronchial and vascular margins [30], suggesting disease progression [22]. Air bronchograms within the consolidation can often be found Consolidation is reported in 11 to $73 \%$ of patients [21], and is seen much more frequently in the elderly than in young patients [22]. Its presence is rare in early-stage and typically occurs in progressive and peak stages [23]. Multiple consolidations are the most common imaging features in the intensive care unit patients [31].

\section{Crazy paving pattern}

Crazy paving pattern is defined as a GGO with superimposed thickened intra- and inter-lobular septa (Figure 1C) [30]. The latter is due to lymphocyte infiltration of small interstitium [32,33]. It occurs in $5 \%$ to $43 \%$ of patients [23,24]. Crazy paving pattern is a common finding in progressive and peak stage of the disease [23].

\section{Vascular enlargement}

Vascular enlargement refers to a segmental or subsegmental pulmonary artery with a diameter $>3 \mathrm{~mm}$ in GGO and consolidation areas (Figure 3). It is a common finding in COVID-19 patients and was reported in 70 to $90 \%$ of patients $[15,35,36]$. This finding is probably related to three processes, in isolation or varying combinations: hyperemia induced by acute inflammatory reaction; small pulmonary embolism, and COVID-19 induced vasculitis [36]. Since this sign was not described in other pneumonia, it may be useful to the identify COVID-19 [36].

\section{Reversed halo sign}

The reversed halo sign (also called atollo sign) refers to a central round or half-moon shape GGO surrounded by a complete or partial ring-like area of consolidation (Figure 4) [30].

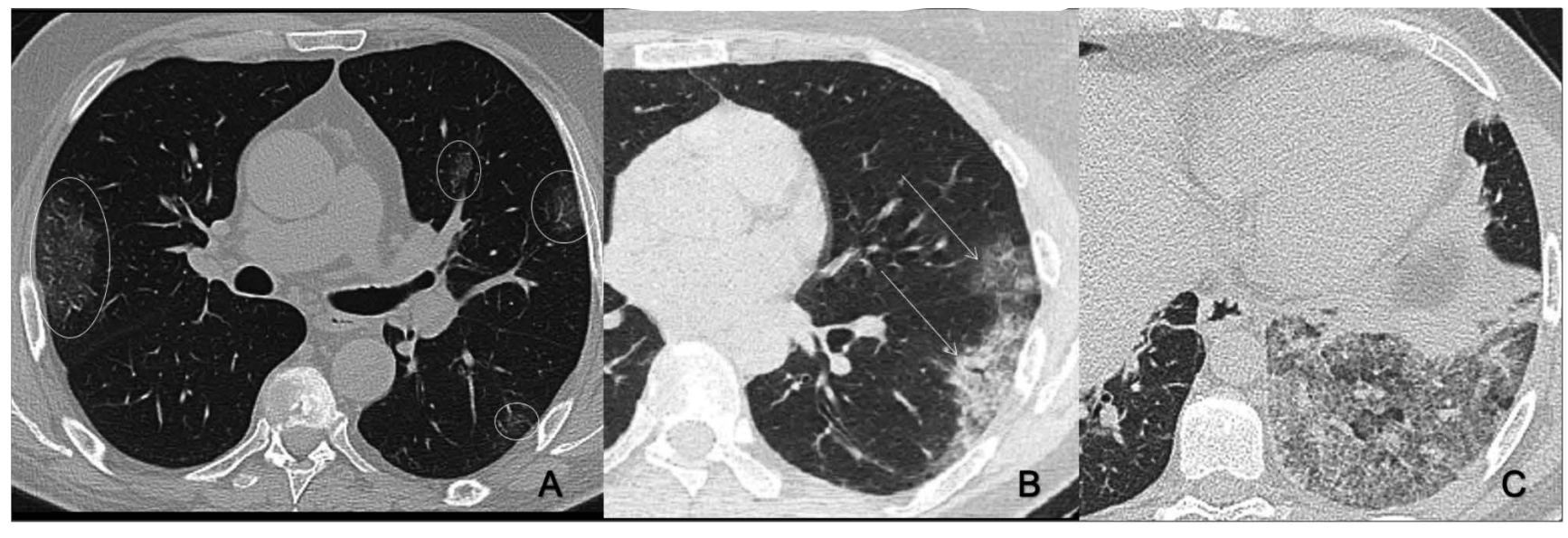

Figure 1. GGO patterns. A) Axial unenhanced CT image obtained in a 66-year-old male shows multiple bilateral patchy ground glass opacities (circles) with predominantly peripheral distribution. B) Axial unenhanced CT image obtained in a 50-year-old female shows ground glass opacities with consolidation in the lower left lung lobe and in the lingula. C) Axial CT image obtained in a 68-year-old female shows a marked crazy paving in the left lower lung lobe. 
The pathological responsible mechanism is the inflammatory reaction, which leads to alveolar septal thickening and cellular debris in the alveoli [37]. This sign is extremely rare in COVID19 patients. Bernheim et al. reported its presence in approximately $2 \%$ in a series of 121 patients [34]. The detection of this sign can suggest a progression from GGO to consolidation or an inflammatory repair $[34,38]$.

\section{Nodule}

A discrete nodule is a rounded opacity, measuring less than 3 $\mathrm{cm}$ in diameter (Figure 5A) [30]. A pure solid nodule is a rare finding in COVID-19 patients. More frequently, a nodule is surrounded by a peripheral halo of GGO, due to diffuse progressive alveolar damage ("halo sign") (Figure 5B) $[33,39,40]$. The halo sign is typically described in early-stage of disease [15,33-40].

\section{Fibrosis}

Fibrosis is a dysregulated repair process to an inflammatory reaction [41]. In case of COVID-19 pneumonia, alveolar epithelial injury can activate fibroblasts and myofibroblasts to repair the damage resulting in lung fibrosis and irreversible injury [32,41]. Fibrosis and strip-like lesions can cause bronchial traction and consequent bronchus irreversible distortion (i.e., bronchiectasis) (Figure 6) [42].

\section{Subpleural curvilinear line}

A subpleural curvilinear line is a curvilinear opacity, 1-3 mm in thickness, parallel to the pleural surface (Figure 7). It may occur for a supine position or may reflect edema or fibrosis process [30].

This finding is reported in approximately $10 \%$ of asymptomatic patients and probably is due to edema or fibrosis $[42,43]$. When it is found in the dependent, posteroinferior portion of the lung, atelectasis is the most likely cause [30].

\section{Pleural changes}

Pleural effusion and pleural thickening more frequently occur in severe COVID-19 patients (Figure 8) [43,44]. In a large meta-analysis, Zhu et al. have reported pleural thickening in $27.1 \%$ of patients and pleural effusion in $5.3 \%$ of patients [45]. Pleural inflammatory reaction can be due to inflammation spread from subpleural lesions and septa [46]. Pleural effusion can also be related to fluid overload due to prior cardiac or renal disease [44].

\section{Hilar and mediastinal lymphadenopathy}

Hilar or mediastinal lymphadenopathy is a lymph node with a short axis greater than $1 \mathrm{~cm}$ (Figure 9) [30]. It is a rare finding and occurs in severe disease $[35,43]$.

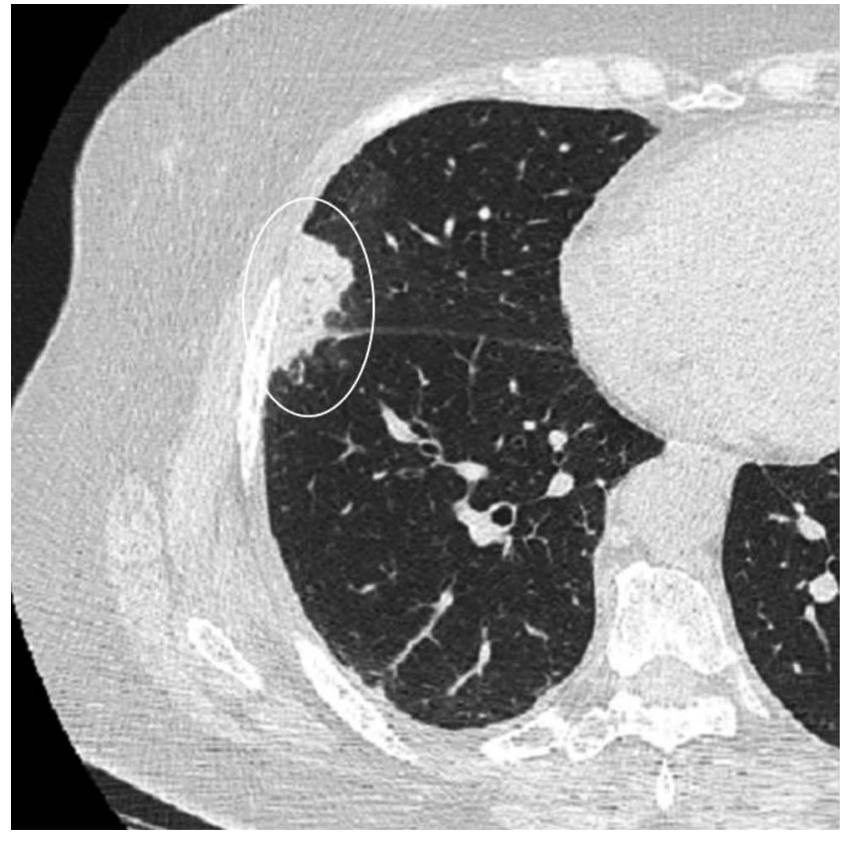

Figure 2. Axial CT image obtained in a 50-year-old female COVID-19 patient shows a subpleural consolidation area (circle) in middle and lower lung lobes.

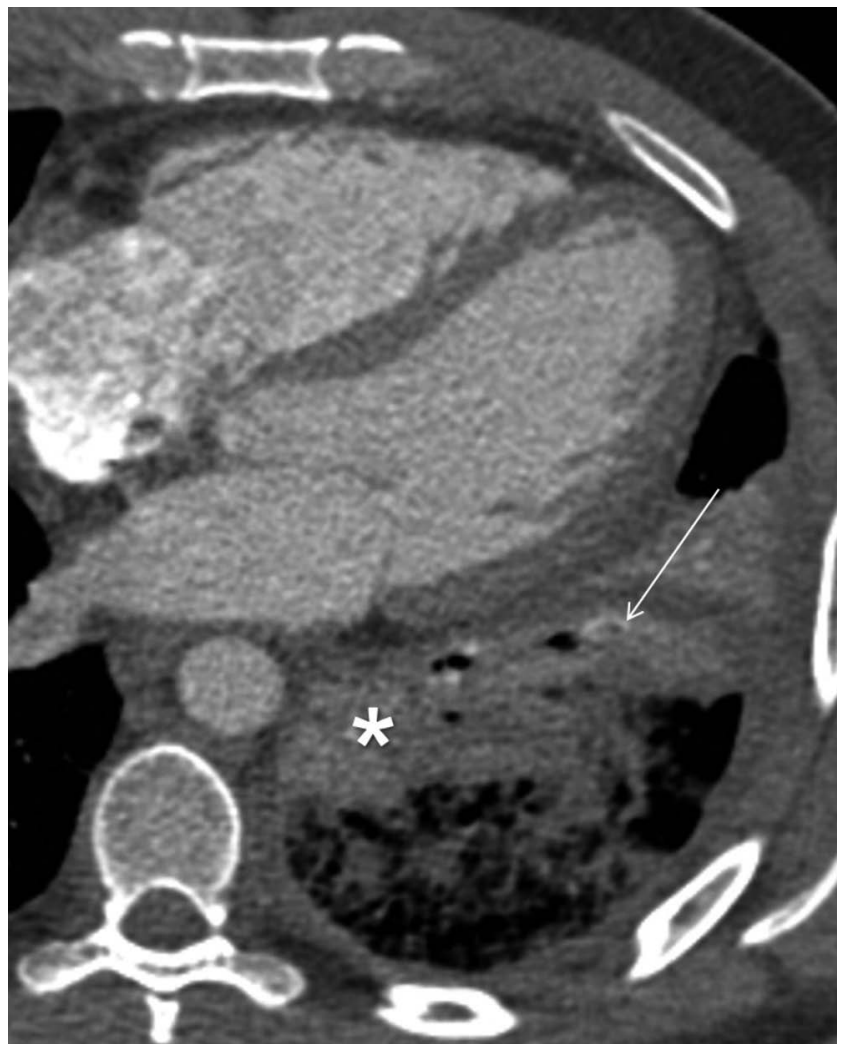

Figure 3. Axial contrast-enhanced CT image obtained in a 33year-old female COVID-19 patient shows vascular enlargement due to a thrombus (arrow) in a large consolidation area (asterisk). 


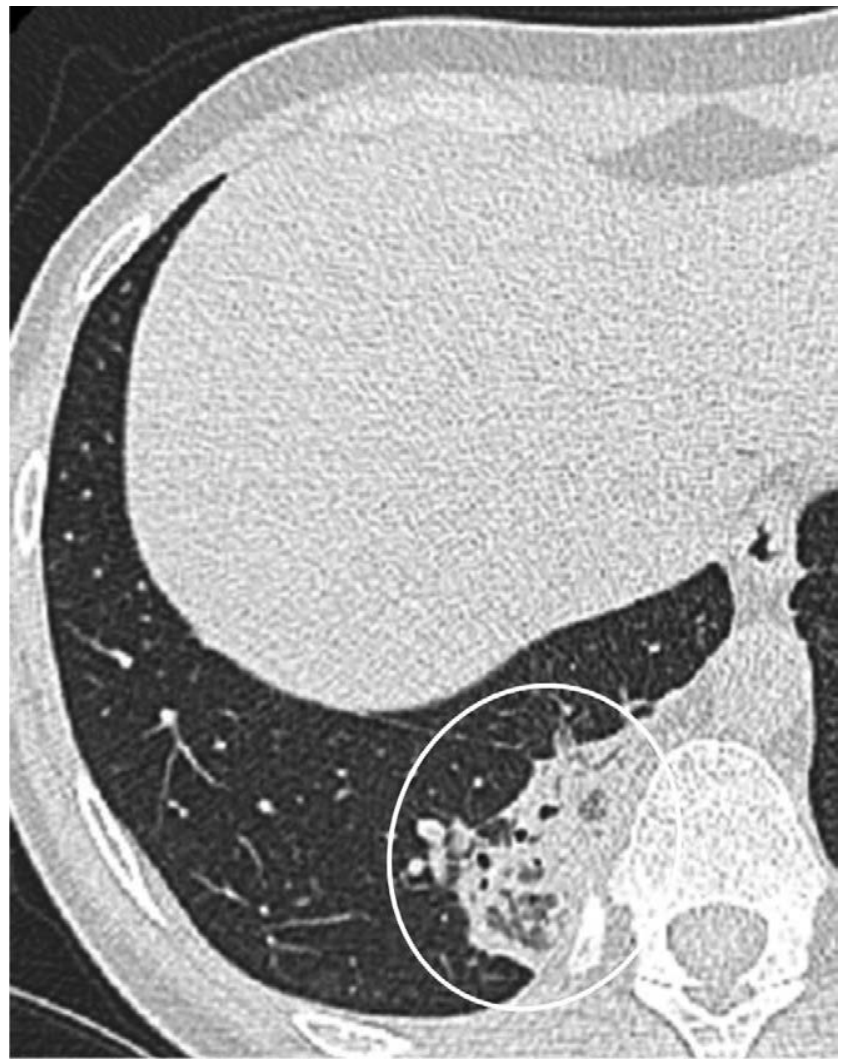

\section{Pericardial effusion}

Pericardial effusion is rare and suggests a severe condition (Figure 8) [35,43]. Of note, the concomitant presence of lymphadenopathies, pleural effusion, and extensive tiny lung nodules raise suspicion of bacterial superinfection [47].

\section{Abdominal findings}

Abdominal manifestations, including gastrointestinal (GI) symptoms (e.g., diarrhea and vomiting) and abnormal liver function are not uncommon and usually occur in severe stage of COVID-19 [48]. Isolated abdominal manifestations at presentation (i.e., without respiratory symptoms) are less frequent [48]. In a meta-analysis including 6064 patients, Mao et al. have found GI symptoms in $15 \%$ of patients, abnormal liver function in $19 \%$ of patients, and isolated GI symptoms in $10 \%$ of patients [48]. The latter has an increased risk of delayed diagnosis of COVID-19 with

Figure 4. Axial CT image obtained in a 23-year-old female COVID-19 patient shows a ground glass area surrounded by a ring-like consolidation area (circle) in the peripheral right lower lobe (reversed halo sign).

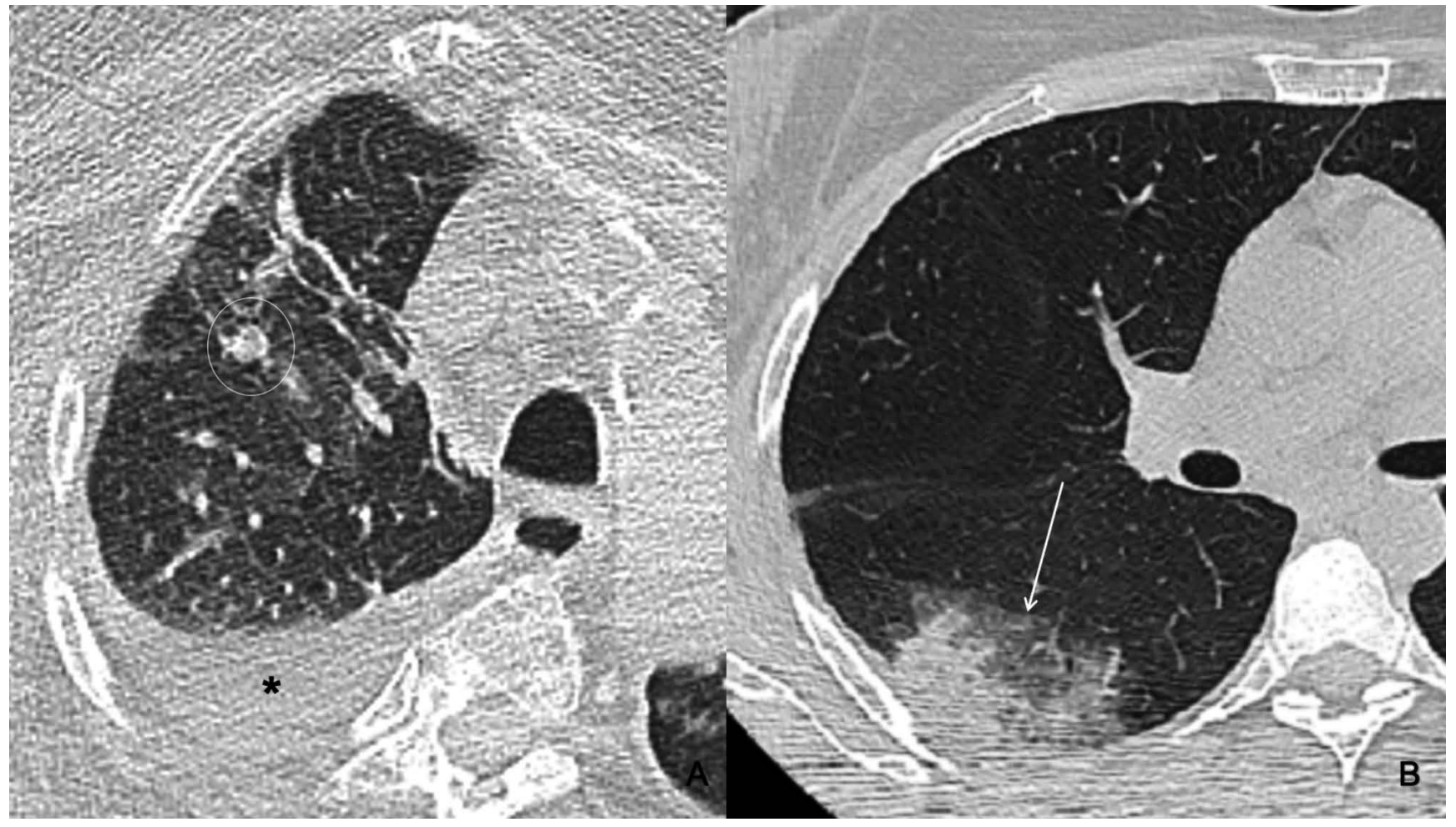

Figure 5. Lung nodule. A) Axial unenhanced CT image obtained in a 61-year-old female shows a nodule (circle) in the right upper lobe. Also note pleural effusion (asterisk). B) Axial CT image obtained in a 61-year-old female COVID-19 patient shows a nodule surrounded by a ground glass halo (arrow) in the peripheral right lower lobe (halo sign). 
the risk of virus-spreading [48]. In this case, the detection of typical findings of COVID-19 pneumonia on the lung bases of abdomen CT scan, can raise the suspicion of COVID-19, despite the absence of respiratory symptoms [49].

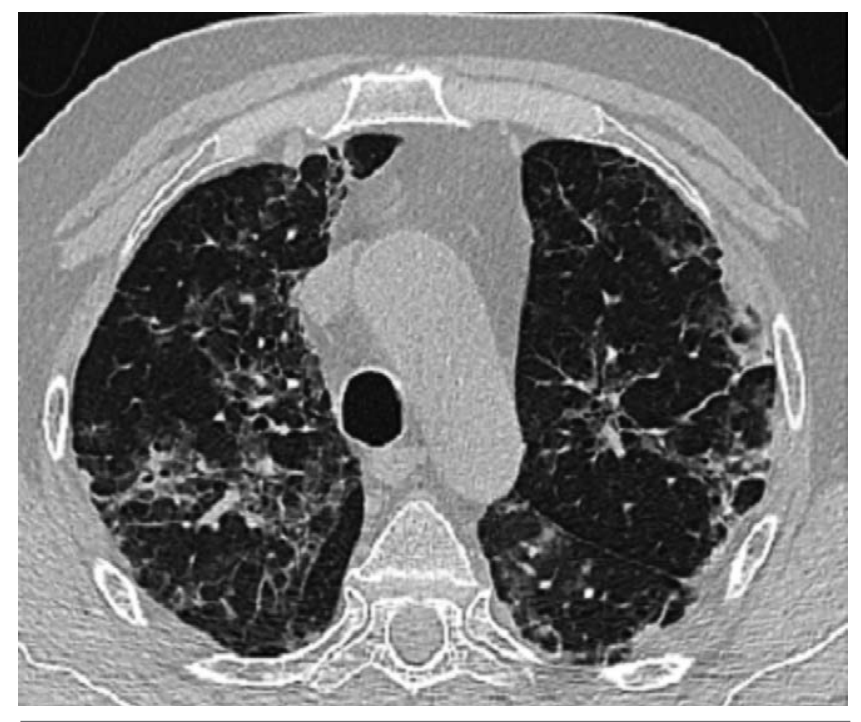

Figure 6. Axial unenhanced CT image obtained in a 68-year-old male COVID-19 patient shows extensive fibrosis and traction bronchiectasis, involving the entire lung parenchyma.

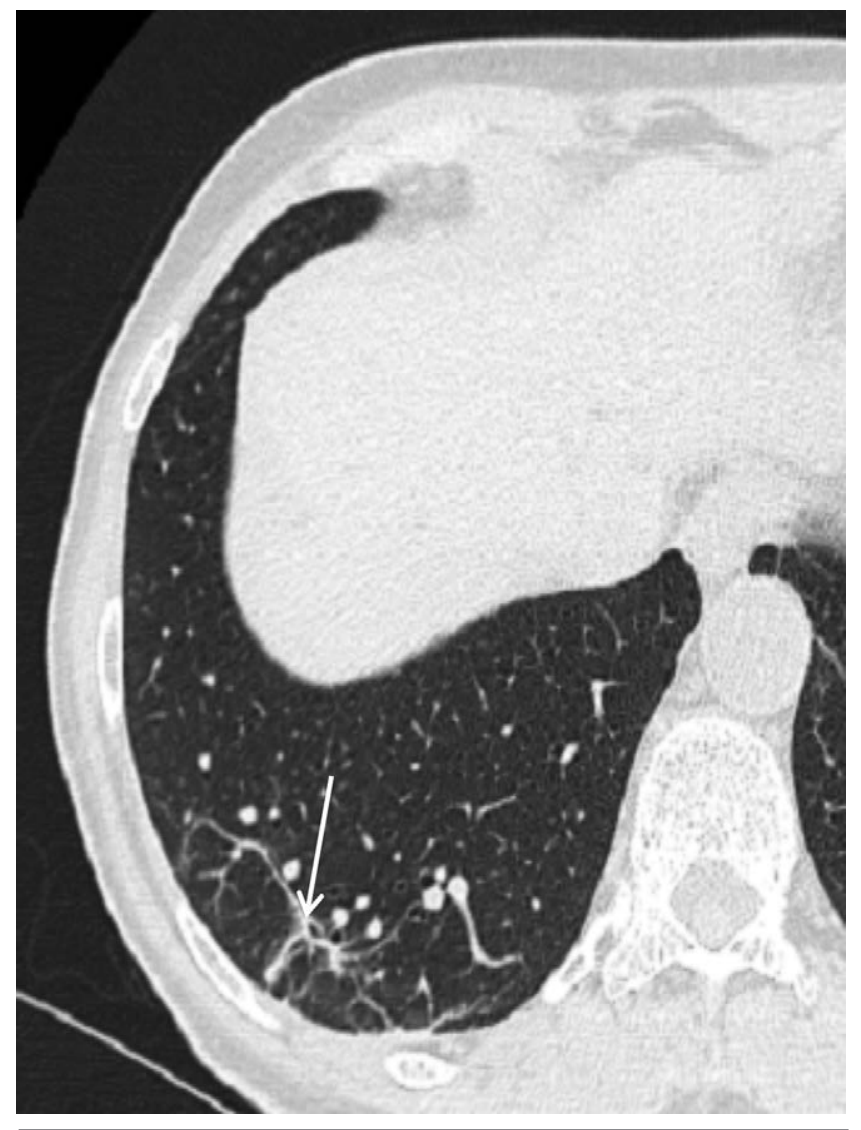

Figure 7. Axial CT image obtained in a 60-year-old male COVID19 patient shows subpleural lines (arrow) in the lower right lobe.
GI and liver involvement can be due to the expression of angiotensin converting enzyme 2 (ACE2), the major receptor of coronavirus or microvascular injury due to inflammation response, that leads to arterial and venous thrombi [50-52].

GI abnormalities are preferentially evaluated at $\mathrm{CT}$ and include bowel wall thickening, pneumatosis, portal venous gas, thrombosis of the portal vein (Figure 10) and upper mesenteric vessels and bowel ischemia $[50,53,54]$.

Ultrasound is most commonly performed for elevated liver enzymes, and can show gallbladder sludge and distension, suggestive of cholestasis [50].

\section{Neurologic findings}

Neurologic symptoms are not uncommon in COVID-19 patients, especially in severe stage of the disease [55-59]. Rarely, they are the only presenting symptoms of COVID-19 [55]. As with abdominal manifestations, the cause of neurologic impairment is

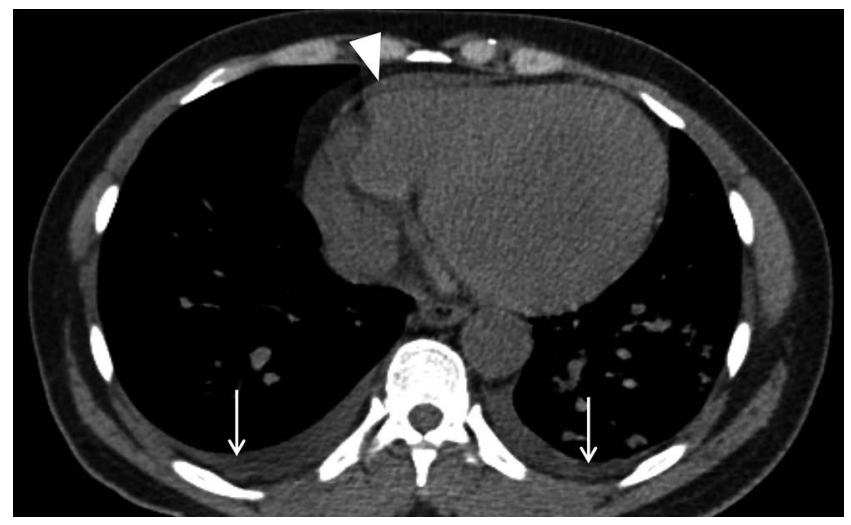

Figure 8. Axial unenhanced CT image obtained in a 40-year-old male COVID-19 patient shows bilateral pleural effusion (arrows) and pericardial effusion (arrowhead).

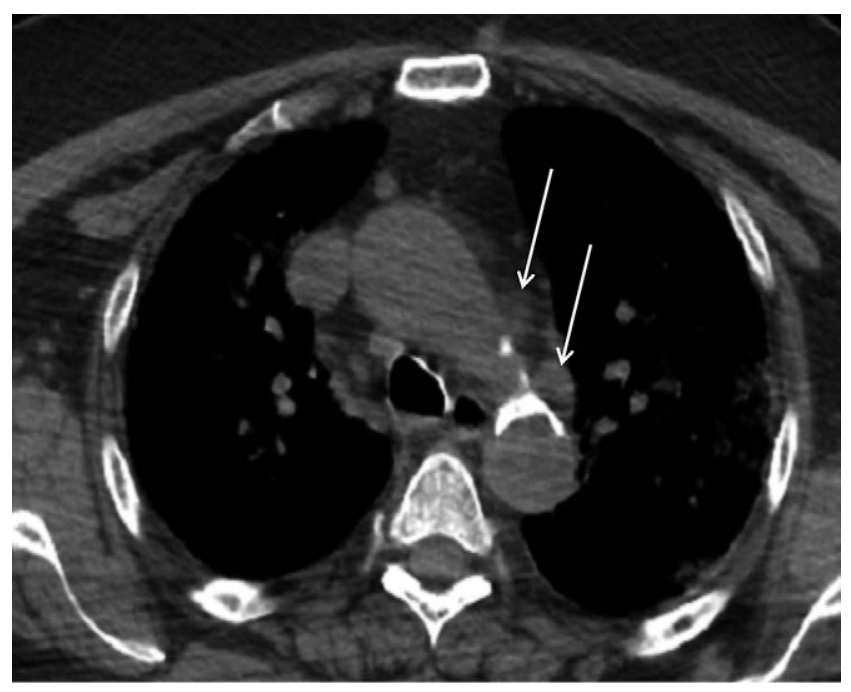

Figure 9. Axial unenhanced CT image obtained in a 69-year-old female COVID-19 patient shows multiple mediastinal lymphadenopathies (arrows). 
ACE2 expression [55]. Main neurological findings are polyneuropathy, encephalopathy, demyelinating lesions, brain microhemorrhage, and ischemic stroke (Figure 11) [55-59]. The latter can be

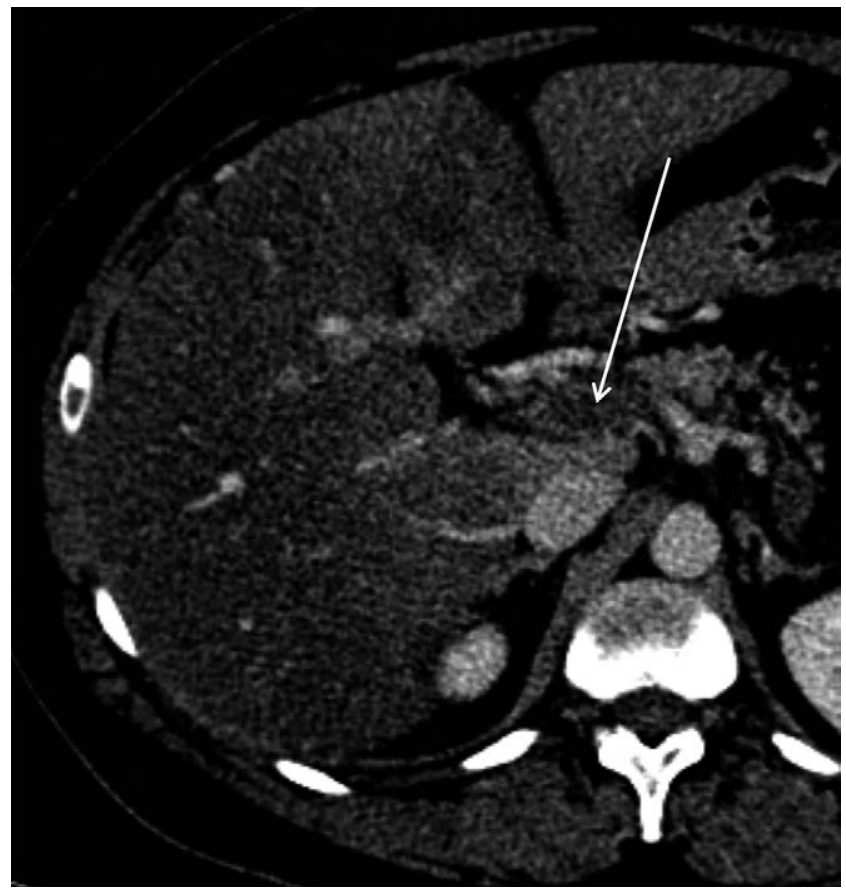

Figure 10. Axial portal venous phase contrast-enhanced CT image obtained in a 40-year-old male COVID-19 patient shows hypoattenuation within mildly dilated thrombosed main portal vein (arrow).

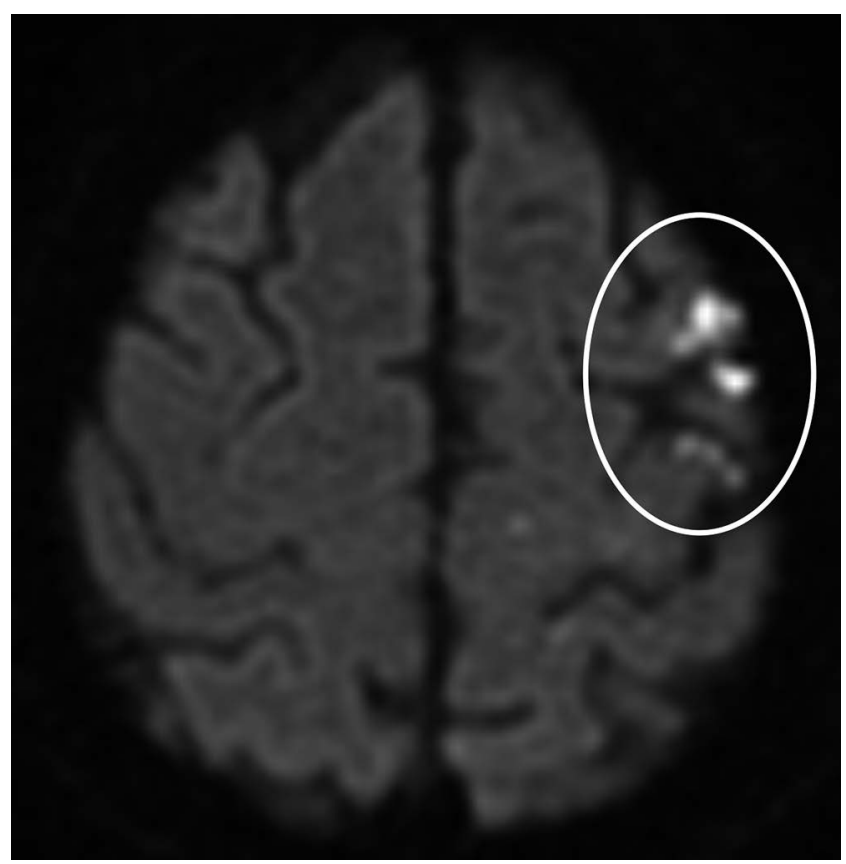

Figure 11. Brain MRI obtained in a 65-year-old male COVID-19 patient shows show multiple acute lacunar ischemic strokes in the left frontal and left parietal lobe (circle) visible as hyperintense areas on diffusion-weighted sequence $\left(b=1000 \mathrm{~s} / \mathrm{mm}^{2}\right)$. related to increased thrombosis rates in coronavirus infection [56] Although imaging findings are not specific of COVID-19, CT and MRI are strongly recommended for the evaluation of neurologic involvement.

\section{Conclusions}

Although RT-PCR is the reference assessment for the diagnosis of COVID-19 infection, imaging plays a crucial role for initial evaluation and follow-up of COVID-19 positive patients and to exclude other disease conditions, which can be treated. Physicians should be familiar with typical and atypical COVID-19 imaging findings.

\section{References}

1. Chan JF-W, Yuan S, Kok K-H, et al. A familial cluster of pneumonia associated with the 2019 novel coronavirus indicating person-to-person transmission: a study of a family cluster. Lancet 2020;395:514-23.

2. Huang C, Wang Y, Li X, et al. Clinical features of patients infected with 2019 novel coronavirus in Wuhan, China. Lancet 2020;395:497-506.

3. Zhu N, Zhang D, Wang W, et al. A novel coronavirus from patients with pneumonia in China, 2019. N Engl J Med 2020;382:727-33.

4. Wang F-S, Zhang C. What to do next to control the 2019-nCoV epidemic? Lancet 2020;395:391-3.

5. World Health Organization. Coronavirus disease (COVID-19) Situation Report - 135. Available from: https://www.who.int/ docs/default-source/coronaviruse/situation-reports/20200604covid-19-sitrep-136.pdf?sfvrsn=fd36550b_2

6. Yang X, Yu Y, Xu J, et al. Clinical course and outcomes of critically ill patients with SARS-CoV-2 pneumonia in Wuhan, China: a single-centered, retrospective, observational study. Lancet Respir Med 2020;8:475-81.

7. Kimball A, Hatfield KM, Arons M, et al. Asymptomatic and presymptomatic SARS-CoV-2 infections in residents of a long-term care skilled nursing facility - King County, Washington, March 2020. MMWR Morb Mortal Wkly Rep 2020;69:377-81.

8. Wang XF, Shi GC, Wan HY, et al. Clinical features of three avian influenza H7N9 virus-infected patients in Shanghai. Clin Respir J 2014;8:410-6.

9. Badawi A, Ryoo SG. Prevalence of comorbidities in the Middle East respiratory syndrome coronavirus (MERS-CoV): a systematic review and meta-analysis. Int $\mathrm{J}$ Infect Dis 2016;49:129-33.

10. Raptis CA, Hammer MM, Short RG, et al. Chest CT and coronavirus disease (COVID-19): A critical review of the literature to date. AJR AJR Am J Roentgenol 2020;215:839-42.

11. Guan W-J, Ni Z-Y, Hu Y, et al. Clinical characteristics of coronavirus disease 2019 in China. N Engl J Med 2020;382:1708-20.

12. Qian M, Yi Q, Qihua F, Ming G. Understanding the influencing factors of nucleic acid detection of 2019 novel coronavirus. Chin J Lab Med 2020;43:213-6.

13. World Health Organization. Infection prevention and control during health care when novel coronavirus $(\mathrm{nCoV})$ infection is suspected. Available from: https://www.who.int/publications/i/ item/10665-331495 
14. Rubin GD, Ryerson CJ, Haramati LB, et al. The role of chest imaging in patient management during the COVID-19 pandemic: A multinational consensus statement from the Fleischner Society. Radiology 2020;296:172-80.

15. Li Y, Xia L. Coronavirus disease 2019 (COVID-19): Role of chest CT in diagnosis and management. AJR Am J Roentgenol 2020;214:1280-6.

16. Mossa-Basha M, Medverd J, Linnau K, et al. Policies and guidelines for COVID-19 preparedness: Experiences from the University of Washington. Radiology 2020;296:E26-31.

17. American College of Radiology . ACR Recommendations for the use of chest radiography and computed tomography (CT) for suspected COVID-19 infection. Available from: https://www.acr.org/Advocacy-and-Economics/ACR-PositionStatements/Recommendations-for-Chest-Radiography-andCT-for-Suspected-COVID19-Infection

18. Chen S-G, Chen J-Y, Yang Y-P, et al. Use of radiographic features in COVID-19 diagnosis. J Chinese Med Assoc 2020;83:644-7.

19. Soldati G, Smargiassi A, Inchingolo R, et al. Is there a role for lung ultrasound during the COVID-19 pandemic? J Ultrasound Med 2020;39:1459-62.

20. Smith MJ, Hayward SA, Innes SM, Miller ASC. Point-of-care lung ultrasound in patients with COVID-19 - a narrative review. Anaesthesia 2020;75:1096-104.

21. Xu B, Xing Y, Peng J, et al. Chest CT for detecting COVID19: A systematic review and meta-analysis of diagnostic accuracy. Eur Radiol 2020;30:5720-7.

22. Song F, Shi N, Shan F, et al. Emerging 2019 novel coronavirus (2019-NCoV) pneumonia. Radiology 2020;295:210-17.

23. Pan F, Ye T, Sun P, et al. Time course of lung changes on chest CT during recovery from 2019 novel coronavirus (COVID-19) pneumonia. Radiology 2020;295:715-21.

24. Han R, Huang L, Jiang H, et al. Early clinical and CT manifestations of coronavirus disease 2019 (COVID-19) pneumonia. AJR Am J Roentgenol 2020;215:338-43.

25. Shi H, Han X, Zheng C. Evolution of CT manifestations in a patient recovered from 2019 novel coronavirus (2019-nCoV) pneumonia in Wuhan, China. Radiology 2020;295:20.

26. Chung M, Bernheim A, Mei X, et al. CT Imaging features of 2019 novel coronavirus (2019-nCoV). Radiology 2020;295:202-7.

27. Ai T, Yang Z, Hou H, et al. Correlation of chest CT and RTPCR testing in coronavirus disease 2019 (COVID-19) in China: A report of 1014 cases. Radiology 2020;296:E32-40.

28. Bai Y, Yao L, Wei T, et al. Presumed asymptomatic carrier transmission of COVID-19. JAMA 2020;323:1406-7.

29. Meng H, Xiong R, He R, et al. CT imaging and clinical course of asymptomatic cases with COVID-19 pneumonia at admission in Wuhan, China. J Infect 2020;81:e33-9.

30. Hansell DM, Bankier AA, MacMahon H, et al. Fleischner Society: Glossary of terms for thoracic imaging. Radiology 2008;246:697-722.

31. Salehi S, Abedi A, Balakrishnan S, Gholamrezanezhad A. Coronavirus disease 2019 (COVID-19): A systematic review of imaging findings in 919 patients. AJR Am J Roentgenol 2020;215:87-93.

32. Xu Z, Shi L, Wang Y, et al. Pathological findings of COVID19 associated with acute respiratory distress syndrome. Lancet Respir Med 2020;8:420-2.

33. Hu Q, Guan H, Sun Z, et al. Early CT features and temporal lung changes in COVID-19 pneumonia in Wuhan, China. Eur J Radiol 2020;128:109017.
34. Bernheim A, Mei X, Huang M, et al. Chest CT findings in coronavirus disease-19 (COVID-19): Relationship to duration of infection. Radiology 2020;295:200463.

35. Caruso D, Zerunian M, Polici M, et al. Chest CT features of COVID-19 in Rome, Italy. Radiology 2020;296:E79-85.

36. Parry AH, Wani AH. Segmental pulmonary vascular changes in COVID-19 pneumonia. AJR Am J Roentgenol 2020;215:W33.

37. Chiarenza A, Esposto Ultimo L, Falsaperla D, et al. Chest imaging using signs, symbols, and naturalistic images: a practical guide for radiologists and non-radiologists. Insights Imaging. 2019;10:114.

38. Farias L de PG de, Strabelli DG, Sawamura MVY. COVID-19 pneumonia and the reversed halo sign. J Bras Pneumol 2020;46:e20200131.

39. Li Y, Xia L. Coronavirus Disease 2019 (COVID-19): Role of chest CT in diagnosis and management. AJR Am J Roentgenol 2020;216:1280-6.

40. Xia W, Shao J, Guo Y, et al. Clinical and CT features in pediatric patients with COVID-19 infection: Different points from adults. Pediatr Pulmonol 2020;55:1169-74.

41. Thannickal VJ, Toews GB, White ES, et al. Mechanisms of pulmonary fibrosis. Annu Rev Med 2004;55:395-417.

42. Zhou S, Wang Y, Zhu T, Xia L. CT features of coronavirus disease 2019 (COVID-19) pneumonia in 62 Patients in Wuhan, China. AJR Am J Roentgenol 2020;214:1287-94.

43. Li K, Wu J, Wu F, et al. The clinical and chest CT features associated with severe and critical COVID-19 pneumonia. Invest Radiol 2020;55:1-5.

44. Yu M, Xu D, Lan L, et al. Thin-section chest CT imaging of coronavirus disease 2019 pneumonia: Comparison between patients with mild and severe disease. Radiol Cardiothorac Imaging 2020;2:e200126.

45. Zhu J, Zhong Z, Li H, et al. CT imaging features of 4,121 patients with COVID $\square$ 19: a meta $\square$ analysis. J Med Virol 2020;92:891-902.

46. Wu J, Pan J, Teng D, Xu X, et al. Interpretation of CT signs of 2019 novel coronavirus (COVID-19) pneumonia. Eur Radiol 2020;30:5455-62.

47. Kanne JP, Little BP, Chung JH, et al. Essentials for radiologists on COVID-19: An update-radiology scientific expert panel. Radiology 2020;296:E113-4.

48. Mao R, Qiu Y, He J, et al. Manifestations and prognosis of gastrointestinal and liver involvement in patients with COVID19: a systematic review and meta-analysis. Lancet Gastroenterol Hepatol 2020;5:667-78).

49. Siegel A, Chang PJ, Jarou ZJ, et al. Lung base findings of coronavirus disease (COVID-19) on abdominal CT in Patients with predominant gastrointestinal symptoms. AJR Am J Roentgenol 2020;215:607-9.

50. Bhayana R, Som A, Li MD, et al. Abdominal imaging findings in COVID-19: Preliminary observations. Radiology 2020;297:E207-158.

51. Chai X, Hu L, Zhang Y, et al. Specific ACE2 expression in cholangiocytes may cause liver damage after 2019-nCoV infection. bioRxiv 2020:2020.02.03.931766.

52. Xiao F, Tang M, Zheng X, et al. Evidence for gastrointestinal infection of SARS-CoV-2. Gastroenterology 2020;158:1831-3.

53. de Barry O, Mekki A, Diffre C, et al. Arterial and venous abdominal thrombosis in a 79-year-old woman with COVID19 pneumonia. Radiol Case Rep 2020;15:1054-7.

54. Beccara LA, Pacioni C, Ponton S, Francavilla S, Cuzzoli A. Arterial mesenteric thrombosis as a complication of SARS- 
CoV-2 infection. Eur J Case Rep Intern Med 2020;7:001690.

55. Mao L, Jin H, Wang M, et al. Neurologic manifestations of hospitalized patients with coronavirus disease 2019 in Wuhan, China. JAMA Neurol 2020;77:683-90.

56. Kandemirli SG, Dogan L, Sarikaya ZT, et al. Brain MRI findings in patients in the intensive care unit with COVID-19 infection. Radiology 2020;297:E232-5.

57. Asadi-Pooya AA, Simani L. Central nervous system manifes- tations of COVID-19: A systematic review. J Neurol Sci 2020;413:116832.

58. Zanin L, Saraceno G, Panciani PP, et al. SARS-CoV-2 can induce brain and spine demyelinating lesions. Acta Neurochir (Wien).2020;162:1491-4.

59. Sachs JR, Gibbs KW, Swor DE, et al. COVID-19-associated leukoencephalopathy. Radiology 2020;296:E184-5. 